\title{
The evolution of neuropsychiatric symptoms during treatment for hyperthyroidism
}

\author{
Oana-Maria NICOLA (MARIOARA) ${ }^{1}$, Alice Elena GHENEA ${ }^{2,3}$, Cristina-Nicoleta VLADOIANU ${ }^{4}$, \\ Valentin CARLIG ${ }^{5}$, Mara CARSOTE ${ }^{6,7}$, Mihaela POPESCU ${ }^{8}$ \\ ${ }^{1}$ Doctoral School, University of Medicine and Pharmacy, Craiova, Romania \\ ${ }^{2}$ Bacteriology-Virology-Parasitology, University of Medicine and Pharmacy, Craiova, Romania \\ ${ }^{3}$ Clinical Emergency County Hospital, Craiova, Romania \\ ${ }^{4}$ Endocrinology, Filantropia Clinical Hospital, Craiova, Romania \\ ${ }^{5}$ Pharmacology, University of Medicine and Pharmacy, Craiova, Romania \\ 6 "Carol Davila" University of Medicine and Pharmacy, Bucharest, Romania \\ ${ }^{7}$ Endocrinology, "C.I.Parhon" National Institute of Endocrinology, Bucharest, Romania \\ ${ }^{8}$ Endocrinology, University of Medicine and Pharmacy, Craiova, Romania
}

\begin{abstract}
Introduction. The link between the action of thyroid hormones and neurological, cognitive and affective function is well known. The aim of the study is to evaluate the changes of hormone's value and symptoms at hyperthyroid patients after 6 months and 1 year of treatment.

Materials and methods. In our study, we enrolled 80 patients with hyperthyroidism (new cases) and we asked them to fill out a questionnaire three times: first time was when they were into a thyrotoxic state (before the antithyroid drug therapy - ATD) and after that during ATD treatment (when they were into an euthyroid state after 6-12 months of treatment, average 7 months).

Results. There is no statistically significant difference between the ages of the study and control groups ( $p$ Student's $t$-test $=0.207>0.005)$, nor between the distributions according to gender ( $p$ Chi square test $=0.827$ ) or area of residence ( $p$ Chi square test $=0.820$ ). For HAMD score, there is highly significant differences $(p<$ 0.001) before treatment, a statistically significant difference after 6 months ( $p$ Student's $t$-test $=0.015<0.05$ ), and no statistically significant difference after 1 year.

Conclusion. Depression and anxiety were improved following thyroid function normalization in hyperthyroidism.
\end{abstract}

Keywords: hyperthyroidism, depression, anxiety, memory loss

\section{INTRODUCTION}

The thyroid gland is involved in maintaining the balance of the human body, whether we are talking about cardiovascular function, immunity, behavior or many other organs and their functions [1].

The link between the action of thyroid hormones and neurological, cognitive and affective function is well known $[2,3]$. Thus, the patients with decreased or increased function of the thyroid gland will present more specific manifestations (for those with hyperthyroidism) and less specific (for those who have hypothyroidism) [2]. These manifestations are reversible and are influenced by the euthyroid status, which is recovered by using substitution therapy [2]. 
Hyperthyroidism is a relatively common disease which also associates some constitutional manifestations [4] that can lead to the severe influence in patients' health. Although the therapy is safe, in current practice there is a tendency to prescribe lower doses than is necessary [4]. Patients with hyperthyroidism require a careful differential diagnosis, because the existing clinical picture in hyperthyroidism mimics the existing one in patients with melancholy, depression and dementia [3]. For hyperthyroidism, the differential diagnosis must include psychosis [5].

The medication for hypothyroidism is prescribed depending on thyroid-stimulating hormone (TSH) values. The recommendation is to administrate levothyroxine if TSH values are $>10 \mathrm{mIU} / \mathrm{l}[6]$. For those with hyperthyroidism, the options of treatment are antithyroid drugs, propylthiouracil (PTU) or imidazole derivatives [7]. High-risk pregnant women, up to 12 weeks of pregnancy, will receive PTU and from the second trimester it will be replaced with methimazole [8].

Together with endocrine diseases, autoimmune, infectios diseases and cancers are the main entities associated with neuro-psychiatric changes [9]. For this patients, if the neuro-pshychiatric symptoms are severe, a correct diagnosis and an effective treatment become a priority [9].

\section{AIM}

The aim of the study is to evaluate the changes of hormone's value and symptoms at hyperthyroid patients after 6 months and 1 year of treatment.

\section{MATERIALS AND METHOD}

\section{Subjects}

In our study we enrolled 80 patients with hyperthyroidism (new cases) and asked them to fill out a questionnaire three times: first time was when they were into a thyrotoxic state (before the antithyroid drug therapy - ATD) and after that during ATD treatment (when they were into an euthyroid state after 6 and 12 months of treatment, average 7 month). The ages of our patients was between 18 and 70 years old. All the patients from our study never took ATD or antidepressant drugs.

We also enrolled 50 healthy patients to compare the results of our study. The exclusion criteria included Mini-Mental State Examination (MMSE) score < 10, Hamilton Anxiety Scale (HAMA) score $>25$, Hamilton Depression Scale (HAMD) score $>25$, addiction to psychoactive substances, neurologic or psychiatric disorders, and other endocrine diseases.

We obtained the informed consent and the approval from all the patients and we applied the principles from the Declaration of Helsinki for our study methods. The experiments we made followed the ethical principles of the assigned institutional board or national committee.The questionnaires contained several measures of psychological factors and they were mailed back to the authors. Of the patients contacted, all signed a consent form.

\section{Tests for neuro-psychiatry assessment}

Each patient had 3 questionnaires: HAMA (Hamilton Anxiety Scale), HAMD (Hamilton Depression Scale) and MMSE (Mini-Mental State Examination).

The Hamilton Scale is used for rating the depression/anxiety and it contains at least 17 questions. Based on the obtained score, between 0 and 7 points - the patient is normal, between 8 and 17 he/she has mild depression/anxiety, between 17 and 25 moderate depression/anxiety and $>25$ severe depression/anxiety [10].

The Mini-Mental State Evaluation (MMSE) is used in the screening of patients with cognitive impairment, in the time-tracking of drug treatments on cognitive performance and in prevention activities through periodic checks that could predict the occurrence of a degenerative disease. A value between 25 and 30 is considered normal, in the interval 21-24 are mild memory loss and under 10 moderate memory loss.

\section{Treatment}

The initial dose of ATD - thiamazole was between 15 to $30 \mathrm{mg} /$ day according to the level of thyrotoxicosis of each patient and the dose of ATD was gradually reduced to maintain a euthyroid state. If after 1 year of treatment, the concentration of FT4 and TSH kept normal values for more than 6 month with $2.5 \mathrm{mg} /$ day thiamazole and TRab(tsh antireceptor antibodies) activity was negative, we stoped the treatment. If after the treatment was stopped, they maintained the serum TSH and FT4 values in the normal range for more than 1 year, excluding relapsed patients, they were considered as being in remission.

\section{Thyroid related tests}

Serum free T4, free T3, TSH ant TRab concentrations were determined by enzyme immunoassay (ECLIA). Normal values for FT4 are between 12 and 22 $\mathrm{pmol} / \mathrm{l}, \mathrm{FT3}$ - 3.4-6.8 pmol/l, TSH 0.27-4.2 $\mu \mathrm{Ul} / \mathrm{ml}$, for TRab - the cut-off limit for clinical decision is 1.75UI/l. We examinated serum FT4, FT3 and TSH concentrations every 1-3 months during ATD treatment for adapting the treatment amount in order to decrease the FT4 and FT3 levels. Serum TRAb activity were examined before treatment, at 6 and 12 months during treatment. 


\section{Follow-up assessments}

We examinated if the treatment had an impact on the depression symptoms and anxiety for our patients after 1 year of treatment with ATD and only after they reached euthyroidism for minimum 3 months. The healthy controls were tested twice, the period of time between the two tests was 12 month. This decision was made so that patients couldn't remember the questions.

\section{Statistical analysis}

Data were recorded using Microsoft Excel files, then it was statistically analyzed to find relationship between clinical and laboratory parameters of patients, then they were processed using the XLSTAT add-on for MS Excel (Addinsoft SARL, Paris, France). To describe the numerical data used in the present study, we used the following statistical indicators: arithmetic mean and standard deviation, and their ratio - the coefficient of variation. We used Student's t test to compare the mean values $( \pm S D)$ of numerical parameters. All comparisons that had $p$-values lower than 0.05 were statistically significant. To analyze the incidence tables for gender and area of residence, we used the Chi-square test $(x 2)$, to show the associations or influences between two categorical factors.

\section{RESULTS}

The data of the age, demographic, hormone's value and scores of HAMA, HAMD, MMSE of healthy controls and the patients with hyperthyroidism before and after treatment are shown in Table 1 and Table 2. There is no statistically significant difference between the ages of the study and control groups ( $p$ Student's $t$-test $=0.207$ $>0.005$ ), nor between the distributions according to gender ( $p$ Chi-square test $=0.827$ ) or area of residence ( $p$ Chi-square test $=0.820$ ).

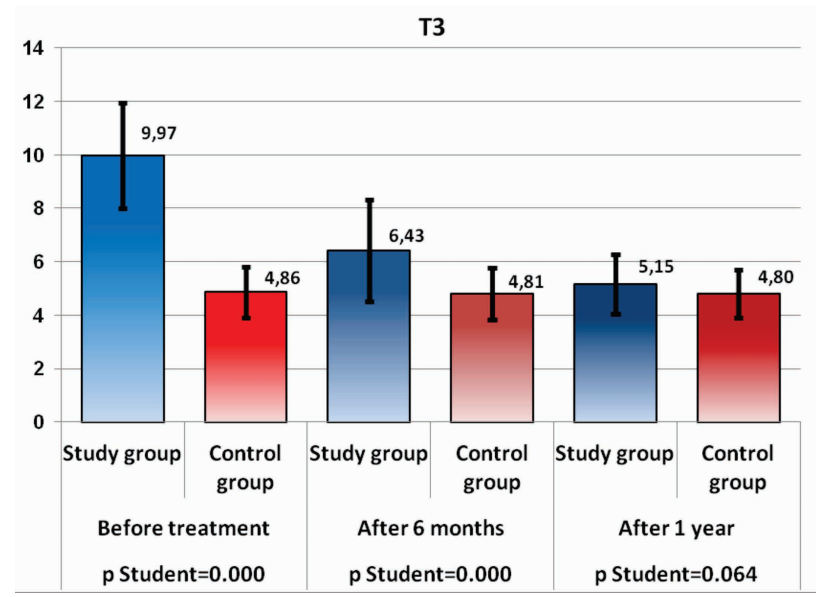

FIGURE 1. T3's value before, after 6 months and after one year of treatment
TABLE 1. Age, demographic, hormone's value and scores of HAMA, HAMD, MMSE of healthy controls and the patients with hyperthyroidism before treatment

\begin{tabular}{|l|l|l|r|}
\hline Before treatment & Study group & Control group & p-value \\
\hline Sex & $21 \mathrm{M} / 59 \mathrm{~F}$ & $14 \mathrm{M} / 36 \mathrm{~F}$ & 0.827 \\
\hline Area & $48 \mathrm{U} / 32 \mathrm{R}$ & $31 \mathrm{U} / 19 \mathrm{R}$ & 0.820 \\
\hline Age & $43.58 \pm 12.73$ & $46.38 \pm 11.49$ & 0.207 \\
\hline FT3 $(\mathrm{pmol} / \mathrm{l})$ & $9.97 \pm 1.97$ & $4.86 \pm 0.96$ & 0.0000 \\
\hline FT4 $(\mathrm{pmol} / \mathrm{l})$ & $33.25 \pm 5.94$ & $16.53 \pm 2.19$ & 0.0000 \\
\hline TSH $(\mu \mathrm{Ul} / \mathrm{ml})$ & $0.02 \pm 0.02$ & $3.10 \pm 0.82$ & 0.0000 \\
\hline TRAb $(\mathrm{UI} / \mathrm{l})$ & $0.97 \pm 0.79$ & $0.94 \pm 0.36$ & 0.7803 \\
\hline HAMD & $10.04 \pm 6.62$ & $3.82 \pm 2.08$ & 0.0000 \\
\hline HAMA & $8.53 \pm 6.45$ & $4.08 \pm 2.21$ & 0.0000 \\
\hline MMSE & $26.46 \pm 5.10$ & $29.32 \pm 0.91$ & 0.0096 \\
\hline
\end{tabular}

TABLE 2. Hormone's value and scores of HAMA, HAMD, MMSE of healthy controls and the patients with hyperthyroidism

\begin{tabular}{|l|l|l|r|}
\hline After 6 months & Study group & Control group & p-value \\
\hline FT3(pmol/l) & $6.43 \pm 1.90$ & $4.81 \pm 0.97$ & 0.0000 \\
\hline FT4 $(\mathrm{pmol} / \mathrm{l})$ & $23.28 \pm 7.86$ & $16.41 \pm 2.18$ & 0.0000 \\
\hline TSH( $\mu \mathrm{UI} / \mathrm{ml})$ & $0.02 \pm 0.02$ & $3.17 \pm 0.78$ & 0.0000 \\
\hline TRAb(UI/l) & $0.84 \pm 0.60$ & $0.94 \pm 0.36$ & 0.2992 \\
\hline HAMD & $6.83 \pm 4.94$ & $3.94 \pm 2.20$ & 0.0002 \\
\hline HAMA & $6.08 \pm 4.82$ & $4.26 \pm 2.40$ & 0.0146 \\
\hline MMSE & $27.28 \pm 4.68$ & $29.20 \pm 1.28$ & 0.4248 \\
\hline After 1 year & Study group & Control group & p-value \\
\hline FT3(pmol/l) & $5.15 \pm 1.11$ & $4.80 \pm 0.90$ & 0.0638 \\
\hline FT4(pmol/l) & $16.68 \pm 3.46$ & $16.55 \pm 2.15$ & 0.8122 \\
\hline TSH( $\mu U I / m l)$ & $1.43 \pm 1.55$ & $3.17 \pm 0.70$ & 0.0000 \\
\hline TRAb(UI/l) & $0.75 \pm 0.42$ & $0.94 \pm 0.36$ & 0.0096 \\
\hline HAMD & $5.46 \pm 3.54$ & $4.04 \pm 2.70$ & 0.0164 \\
\hline HAMA & $5.29 \pm 3.62$ & $4.60 \pm 3.21$ & 0.2737 \\
\hline MMSE & $28.59 \pm 2.37$ & $29.18 \pm 1.27$ & 0.7162 \\
\hline
\end{tabular}

On the other hand, for T3, there are highly significant differences $(p<0.001)$ before treatment and after 6 months, and no statistically significant difference after 1 year ( $p$ Student's t-test $=0.064>0.05)$ (Figure 1$)$.

For T4, there are highly significant differences $(p<0.001)$ before treatment and after 6 months, and no statistically significant difference after 1 year ( $p$ Student's t-test $=0.812>0.05$ ) (Figure 2).

For TSH, there are highly significant differences $(p<0.001)$ at all moments (Figure 3$)$.

For TRAB, there are no significant differences before treatment $(p=0.78>0.05)$ and after 6 months $(p=0.299>0.05)$, and a statistically significant difference after 1 year ( $p$ Student's t-test $=0.010<0.05$ ) (Figure 4).

As for changes of depressive personality (HAMD), anxiety (HAMA) and memory loss (MMSE) between before and during treatment (after 6 month and 1 year) they occur in the next figures.

For HAMA score, there are highly significant differences $(p<0.001)$ before treatment and after 6 months, 
T4

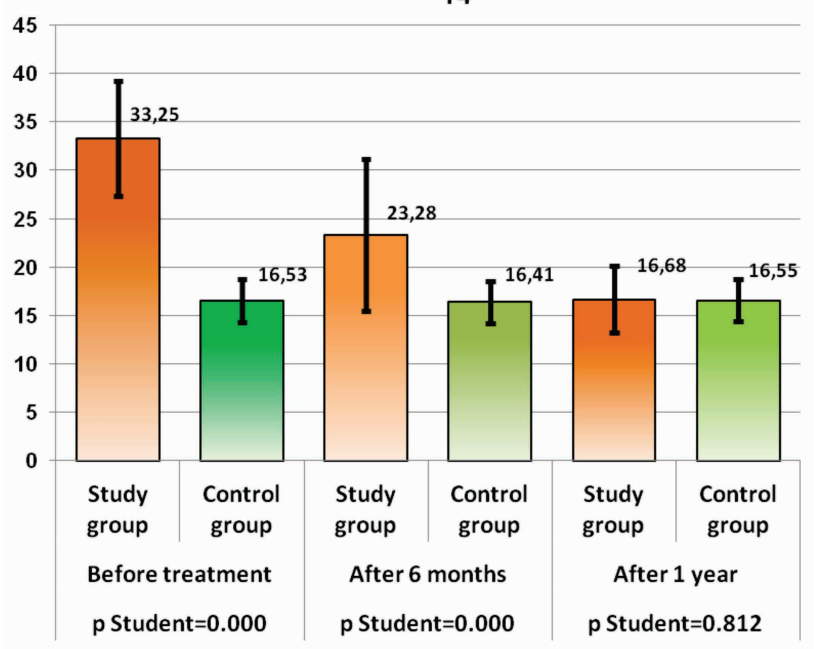

FIGURE 2. Variation of T4 during the treatment with ATD after 6 and 12 months

TSH

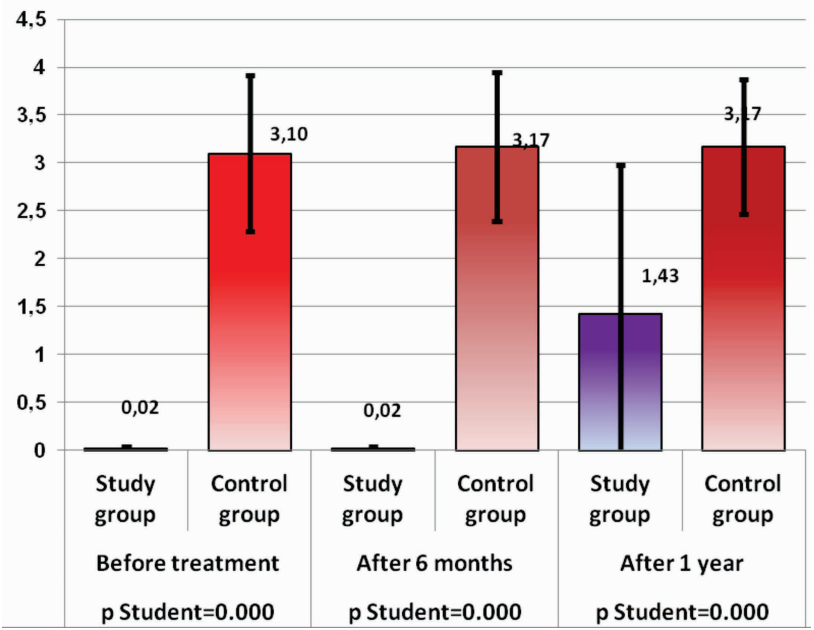

FIGURE 3. TSH's value before, after 6 months and after one year of treatment

TRAb

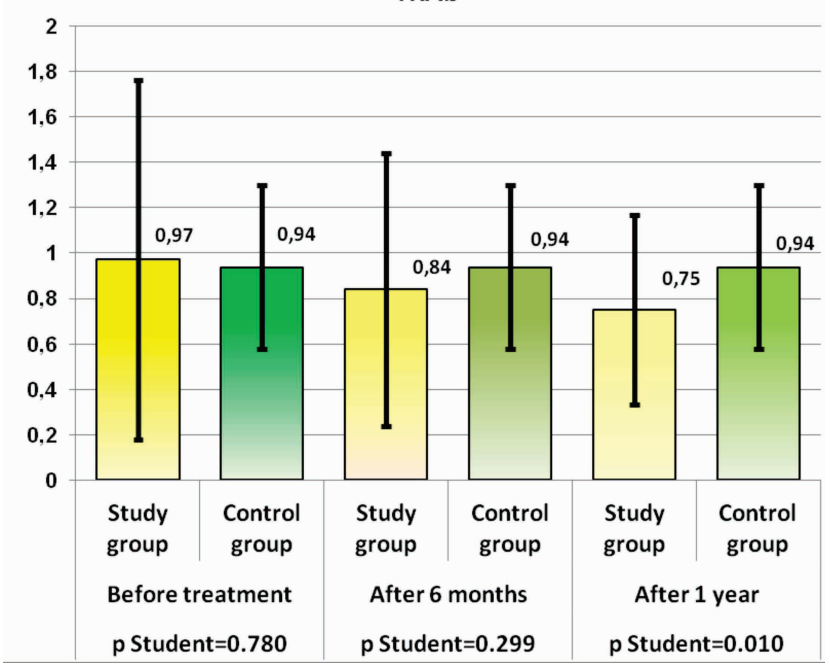

FIGURE 4. Variation of Tsh antireceptor antibodies after treatment

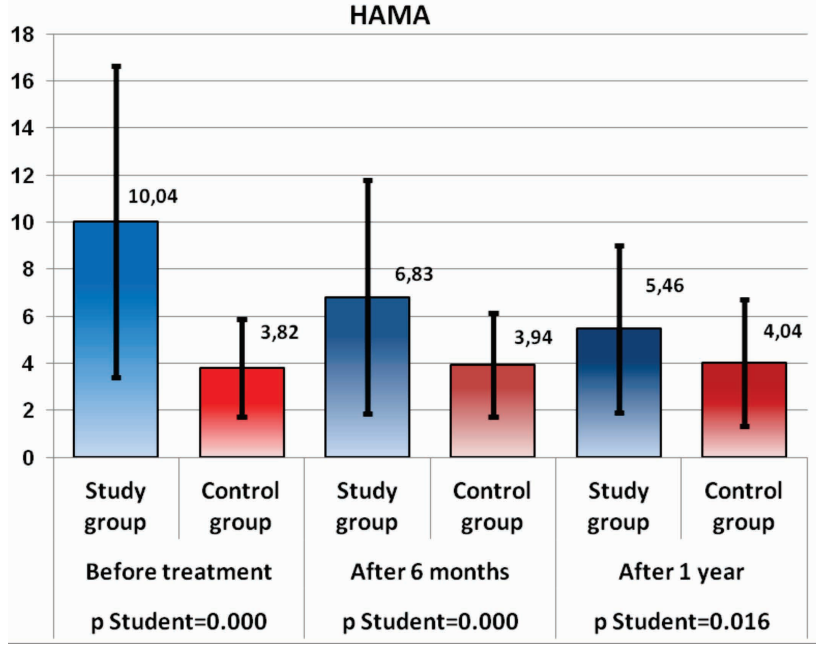

FIGURE 5. Anxiety during the treatment

HAMD

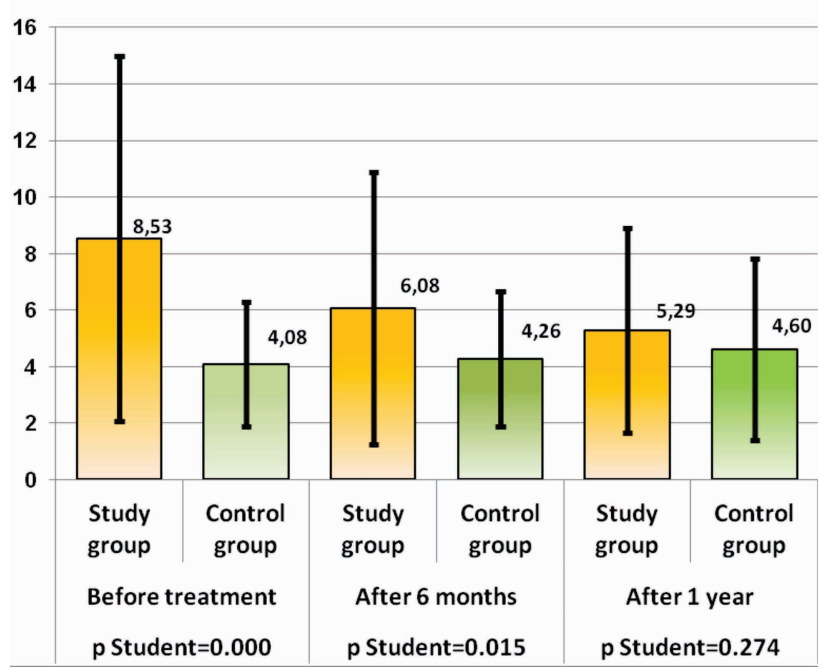

FIGURE 6. Depression variability after 6 and 12 months of treatment

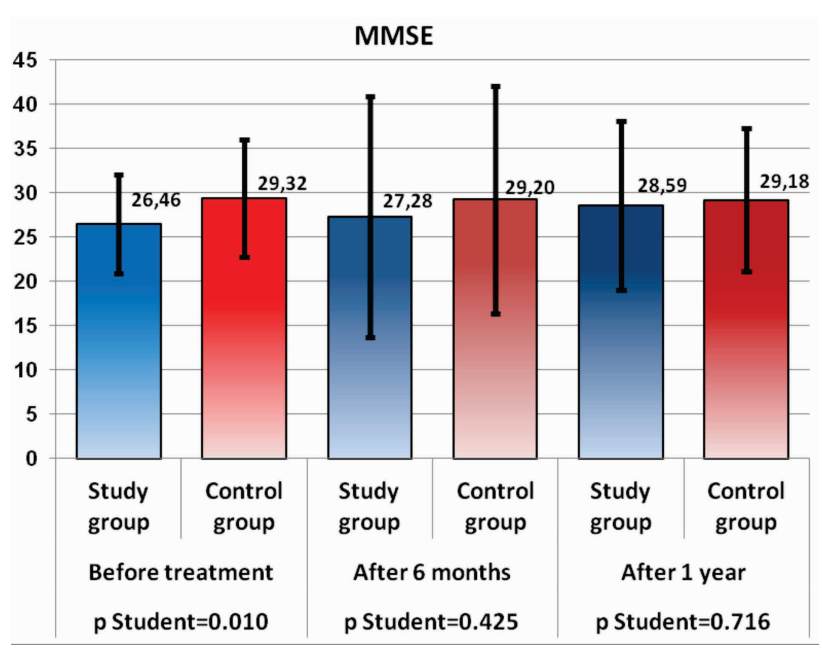

FIGURE 7. Memory loss modification after ATD 
and a statistically significant difference after 1 year ( $p$ Student's t-test $=0.016<0.05$ ) (Figure 5) .

For HAMD score, there is highly significant differences $(p<0.001)$ before treatment, a statistically significant difference after 6 months ( $p$ Student's t-test = $0.015<0.05$ ), and no statistically significant difference after 1 year ( $p$ Student's t-test $=0.274>0.05$ ) (Figure 6) .

For MMSE score, there is significant differences ( $p$ Student's t-test $=0.015<0.05$ ) before treatment, and no statistically significant difference after 6 months ( $p$ Student's t-test $=0.425>0.05)$, and after 1 year ( $p$ Student's t-test $=0.716>0.05)$ (Figure 7 ) .

\section{DISCUSSION}

In our study, as it seen in some others [11], we observed that anxiety and depression scores on Hamilton scale were higher than those of healthy controls. Also, after the treatment, the analysed serum levels was significantly decreased at 6 months and 1 year. Regarding the memory loss, this wasn't significantly modified after treatment, at 6 months and 1 year.

On the other hand, in terms of hyperthyroidism, especially in Basedow Graves disease, it seems that mental disorders such as mania, depression, nervousness, anxiety predominate [12]. These psychiatric disorders are caused by the thyrotoxic condition and may resolve after treatment because thyroid hormones have beta adrenergic effect. In fact, it appears that in small studies anxiety and depression improved after administration of a single thyroid drug, while in large cohorts they persisted in the euthyroid state after treatment $[13,14]$.

In another study [15], symptoms such as rational thinking and feelings sharing were remitted relatively quickly to those treated with antiroids. Psychotherapy can be very helpful in improving the progression of hyperthyroid patients [16].

In patients receiving lithium, thyroid hormones have been shown to influence the cognitive side effects of antidepressant treatment. Lithium has important effects on the thyroid such as thyroid hormone modification (increases TSH and decreases T3, T4). Therefore, the administration of thyroid hormones improves cognitive function in those treated with lithium-based antidepressants. So many patients who could not be given such antidepressants may now be eligible if hormone supplements are given at the same time [17].

After the normalization of thyroid hormones from hyperthyroidism, the reversibility of affective symptoms and attention deficit was also observed [11]. As described by Vogel, there is a significant decrease in affective symptoms when thyroid function is normalized [18].

Fahrenfort et al. have found that after regular treatment in patients with hyperthyroidism, neuro-psychiatric symptoms may resolve, although some patients remain with manifestations even at 1 year of euthyroidism [19].

Triiodothyronine (T3) is often associated with antidepressant treatment to potentiate the response to treatment in the unresponsive [20].

There is no clear evidence regarding the indications for treatment in subclinical thyroid pathology, so in subclinical hyperthyroidism the indication is cardiovascular risk, and in subclinical hypothyroidism there would be progression to overt hypothyroidism [21].

\section{CONCLUSIONS}

Depression and anxiety were improved following thyroid function normalization in hyperthyroidism. In our study, the anxiety and depression's severity decreases after treatment with ATD, which demonstrate the influence of the thyroid hormones in the pathological mecanism of them.

Regarding the memory loss, there were no representative changes, which is why further studies are needed to demonstrate whether excess thyroid hormones influence it if persists for a long time.

The combination of thyroid treatment and antidepressants can be a real help in thyroid pathology, from a clinical point of view, but especially economically speaking.

\section{REFERENCES}

1. Albi E, Kruger M, Hemmersbach R, et al. Impact of gravity on thyoid cells. Int J Mol Sci. 2017;18(5):972.

2. Feldman Z, Shresha R, Hennessey J. Neuropsychiatric manifestations of thyroid disease. Endocrinol Metab Clin North Am. 2013;42(3):453-476.

3. Davis J, Thermont T. Neuropsychiatric aspects of hypothyroidism and treatment reversibility. Minerva Endocrinol. 2007; 32(1):49-65.

4. Roberts $C$, Landenson P. Hypothyroidism. Lancet. 2004;363(9411):793-803.

5. Desai D, Zahedpour Anaraki S, Reddy N, et al. Thyroid Storm Presenting as Psychosis. $J$ Investig Med High Impact Case Rep. 2018;6:23-24.
Conflict of interest: none declared Financial support: none declared 
8. Kobaly K, Mandel S. Hyperthyroidism and pregnancy. Endocrinol Metab Clin North Am. 2019;48(3):533-545.

9. Isaac M, Larson E. Medical conditions with neuropsychiatric manifestations. Med Clin North Am. 2014;98(5):1193-208.

10. Scala de depresie Hamilton [Online]. Available at: www.suflet-linistit.ro/scala-dedepresie-hamilton/.

11. Yuan L, Zhang Y, Luan D, et al. Reversible Affective Symptoms and Attention Executive Control Network Impairment Following Thyroid Function Normalization in Hyperthyroidism. Neuropsychiatr Dis Treat. 2019:15:3305-3312.

12. Pollock MA, Sturrock A, Marshall K, et al. Thyroxine treatment in patients with symptoms of hypothyroidism but thyroid function tests within the reference range: randomised double blind placebo controlled crossover trial. BMJ. 2001;323(7318):891-5.

13. Bommer M, Eversmann T, Pickardt R, et al. Psychological and neuropsychological symtoms in patients with subclinical and remitted hyperthyroidism. Klin Wochenschr. 1990;68:552-558.

14. Fahrenfort JJ, Wilterdink AML, van der Veen EA. Long-term residual complaints and psychological sequate after remission of hyperthyroidism. Psychoneuroimmunology. 2000;25:201-211.

15. Fukao A, Takamatsu J, Kubota S, et al. The thyroid function of Graves' disease patients is aggravated by depressive personality during antithyroid drug treatment. Biopsychosoc Med. 2011;5:9.

16. Fukao A, Takamatsu J. The role of psychological factors on the onset and clinical course of hyperthyroid Graves disease. Recent Res Devel Endocrinol. 2002;3:369-376.

17. Tremont G, Stern RA. Use of thyroid hormone to diminish the cognitive side effects of psychiatric treatment. Psychopharmacol Bull. 1997;33(2):273-280.
18. Vogel A, Elberling TV, Hording M, et al. Affective symptoms and cognitive functions in the acute phase of Graves thyrotoxicosis. Psychoneuroendocrinology. 2007; 32(1):36-43.

19. Fahrenfort JJ, Wilterdink AM, van der Veen EA. Long-term residual complaints and psychosocial sequelae after remission of hyperthyroidism. Psychoneuroendocrinology. 2000;25(2):201-211.

20. Cooper-Kazaz R, Lerer B. Efficacy and safety of triiodothyronine supplementation in patients with major depressive disorder treated with specific serotonin reuptake inhibitors. Int J Neuropsychopharmacol. 2008,11(5):685-699.

21. Franklyn JA. Subclinical thyroid disordersconsequences and implications for treatment. Ann Endocrinol. 2007, 68(4):229-230. 